\title{
Predictors of Suicide Ideations in Adolescents
}

\author{
Rupan Dhillon ${ }^{1}$, Nishtha Mehra ${ }^{2}$ \\ ${ }^{1}$ Assistant Professor, Department of Psychology Guru Nanak Dev University Amritsar. \\ ${ }^{2}$ Scientist 'B', Selection Centre North, Kapurthala \\ Corresponding author: Dr. Rupan Dhillon \\ Email-rupan.oberoi@rediffmail.com
}

\begin{abstract}
Background and Objectives: Adolescence is a crucial phase of one's life, given the physical and psychological changes that occur during this phase. Number of adolescent suicides are on rise and a large number of adolescents report higher suicidal ideations during this phase. Suicide ideation involves thoughts and cognitions about suicidal behavior. It involves thoughts related to desire, intent or method of committing suicide. Research on suicide ideation has established strong links between depression and hopelessness with suicidal thoughts and tendencies. Depressed adolescents are reported to be highly vulnerable to develop suicide ideations. In addition to depression and hopelessness, familial variables like support from parents and emotional bonding with them is detrimental to such outcomes like suicide ideation. The present study thus aims to find depression, hopelessness, emotional autonomy and hardiness as predictors of suicide ideation in adolescents.

Methods: A sample of 400 adolescents $(242=$ Females and $158=$ Males $)$ in the age group of $15-22$ (Mean Age $=18.36$ years) years was taken. Beck Depression Inventory, Beck Hopelessness Scale, Personal Views Survey, Emotional Autonomy Scale and Beck Scale for Suicide Ideation were used to collect the data. The data so obtained for the predictor variables was first factor analysed so as to obtain independent factors.

Results: Results revealed single factor with high factor loadings on all the four predictor variables. The factor scores from this factor were further used in linear regression in order to predict suicide ideation in adolescents. Regression analysis revealed that the factor was a significant predictor of suicide ideation. The obtained results have been elucidated.

Conclusion: There is a very high prevalence of depression, anxiety and stress among interns working in Maharashtra which needs to be effectively dealt with on an urgent basis. The strong association of the prevalence of psychological illnesses with many factors and stressors faced by them will help us highlight the contributory factors and suggest strategies to deal with them effectively at a personal, institutional and national level.
\end{abstract}

Keywords: Suicidal ideation, adolescents, anxiety, depression, emotional autonomy.

(Paper received $-9^{\text {th }}$ July 2018, Peer review completed $-20^{\text {th }}$ July 2018)

(Accepted $-24^{\text {th }}$ August 2018)

\section{INTRODUCTION}

Adolescence is considered a crucial period in the life span of an individual. It is a phase of turmoil and conflicts. It is also called a transitional period where adolescents are developing their identity and gaining autonomy. Lerner and Galambos [1] have described adolescence as a phase of life from 12 to 22 years, beginning in biology and ending in society. It is a transitional and a developmental period in which there is a redefinition and reorganisation of family relations [1]. 
Suicide ideation refers to the presence of self-destructive thoughts. There is an occurrence of thoughts about self-destructive behaviour, however, death may or may not be intended. The thoughts may occupy a person's mind for a moment or may keep recurring. These thoughts may be vague or may involve making of some concrete plans to commit suicide. It may be a harmless thought as many adolescents face emotional problems and conflicts and hence may just develop a thought which maybe transient in nature. However, it may be possible that this thought may become a nightmare.

O Carroll and others defined suicide ideation as "self-reported thoughts of carrying out suicide related behaviour" [2]. Suicidal ideation can be expressed in many ways and also reflects many tendencies to act. People are discontent with their living conditions or it may act as a warning signal for individuals in situations which are perceived as stressful. It may also be a mental preparation for the act. It reflects hopes and illusions about the future. It reflects evaluations about the quality of life which co vary with life events and changing conditions of living. It reflects threats people experience to their self-esteem. It is to be understood that such people have long term vulnerable self-esteem which becomes manifest in times of interpersonal conflict [3].

The interpersonal-psychological theory of suicidal behaviour [4] proposes that an individual will not die by suicide unless he has both the desire to die by suicide and the ability to do so. The theory asserts that when people hold two specific psychological states in their minds simultaneously, and when they do so for long enough, they develop the desire for death. The two psychological states are perceived burdensomeness and a sense of low belongingness or social alienation.

Perceived burdensomeness is the view that one's existence burdens family, friends and society. This view produces the idea that "my death will be worth more than my life to family, friends and society. This view is considered as a fatal misperception. The elevated likelihood of developing perceptions of burdensomeness on others is the common thread between family conflict, unemployment, and physical illness that can account for the associations with suicide [5].

A low sense of belongingness is the experience that one is alienated from others, not an integral part of a family, circle of friends, or valued group. It is said to be a multidimensional construct having various facets. An individual experiencing the mental state of thwarted belongingness might express the loneliness component of the construct by stating, "I did not have a satisfying social interaction today". The second facet of thwarted belongingness is the absence of reciprocally-caring relationships. The Interpersonal Theory includes the assumption that thwarted belongingness is a dynamic cognitive-affective state, rather than a stable trait, that is influenced by both interpersonal and intrapersonal factors [6].

\section{Predictors of Suicide Ideations}

\section{Depression and Hopelessness}

Literature on youth suicidal ideation is linked to depression and feelings of hopelessness [7] and interaction of depression and hopelessness is the strongest predictor of suicide completion [8]. The hopelessness theory [7] and the Cognitive Distortion Theory [9] state that hopelessness is the primary predictor of suicide, even better than depression. The problem with such individuals is that they are unable to interpret events correctly. There are shifts in pattern of thinking, individuals become preoccupied with problems, lose perspective and see suicide as the only solution. These are irrational evaluations of events. They develop a pessimistic belief that their situation will not change.

A study showed that severity of depressive symptoms was the strongest predictor of suicide ideations in the high risk adolescent community sample [10]. In another study of adolescent psychiatric inpatients, depressed mood, negative automatic thoughts and hopelessness were the strongest predictors of suicidal ideations [9].

\section{Emotional Autonomy}

Crittenden [11] has defined emotional autonomy as capacities for taking responsibility of one's own behaviour, making decisions regarding one's life and maintaining supportive relationships. Autonomy is not the same as rejection, alienation or physical separation from parents. Steinberg and Silverberg [12] gave the term "emotional autonomy" to describe the affective disengagement of the adolescent from his or her parents and established a scale to measure it. This term according to them suggests a detachment from parents, which means withdrawing from the family and moving to wider community [11]. 
Emotional autonomy may assume the feature of separation throughout psychological individuation or may take the shape of detachment and maybe characterized by alienation and distrust towards parent's role [12]. In such cases, both as separation and detachment, emotional autonomy plays a role in predicting depression or psychological maladjustment or internalizing problem behaviours [13-14]. Detachment would be related to adolescent's depression. Pace and Zapulla found a significant relationship between detachment and separation and suicide ideation [15].

\section{Hardiness}

The construct of hardiness introduced by Kobasa [16] is a resiliency resource which allows an individual to exhibit certain behaviours which buffer the impact of stress [16]. This construct is composed of three basic, interrelated elements: commitment, control and challenge. These are separate, yet interdependent components which are representative characteristics of a person who embodies an existentialist mode of being and strives for authenticity. Hardy individuals tend to regard new experiences as challenging and exciting rather than disruptive and threatening. Hardy individuals have a sense of commitment to their activities and have a feeling of sufficient control over personal resources to steer life in a wanted direction. This unique style of appraising stressors positively and the belief in their efficacy buffers them from detrimental effects of stress. Hardiness may constitute a resilience factor for adolescent samples, especially for individuals with suicidal ideations [17].

\section{Objective of the Study}

Keeping in mind the theoretical perspectives of suicide ideations and the important predictors of depression, hopelessness, emotional autonomy and hardiness, this study is going to aim to understand the importance of these variables as associated with suicide ideations. This study is designed to study the psychological determinants of suicidal ideations in the adolescent youth.

\section{Hypotheses}

1. The variables of depression, hopelessness, emotional autonomy and hardiness will predict suicide ideations in adolescents.

2. Depression, hopelessness and emotional autonomy will be positively associated with suicide ideations.

3. Hardiness will be negatively associated with suicide ideations.

\section{METHODOLOGY}

\section{Sample}

The adolescents in this study were taken within the age group of 15 -22 years. There are 242 females and 158 males in the sample. The total sample consists of 400 adolescents. The mean age of males is 18.36 with a standard deviation of 1.66 and the mean age of females is 19.14 with a standard deviation of 1.76. Incidental sampling technique is employed for the purpose of the study.

\section{Psychological Tests}

The various tests employed in this study are:

1) Beck Depression Inventory (BDI-II) [18].

2) Beck Hopelessness Scale (BHS) [19].

3) Personal Views Survey (PVS) [20].

4) Emotional Autonomy Scale (EAS) [21].

5) Beck Scale for Suicide Ideation [22].

\section{Beck Depression Inventory (BDI-II)}

It is one of the most popular and widely used depression inventory which can be used in individuals aged 13 and above. BDI-II is a 21 multiple choice question-based inventory that measures various aspects of depression in an individual like guilt, hopelessness, helplessness, weight loss, etc. The answers are scaled 
from 0 to 3 . Scores from 0 to 13 indicates minimum depression, from 14 to 19 is mild depression, 20 to 28 indicates moderate depression and finally individuals falling in the range 29-63 are said to be having severe depression. The reliability of the test stands at $r=0.93$ while the internal consistency is on higher side i.e. $\alpha$ $=0.91$ as suggested by the test maker [18].

\section{Beck Hopelessness Scale (BHS)}

It is a 20 -item self-report inventory that was designed to measure negative attitudes about the future as perceived by adults. It measures three major aspects of hopelessness: feelings about the future, loss of motivation, and expectations. The test is designed for adults, age 17-80. The internal reliability coefficients are reasonably high (Pearson r .82 to .93 in seven norm groups) [19].

\section{Personal Views Survey (PVS)}

This is one of the most important scales used to measure psychological hardiness in individuals. This scale consists of 12 items. The scale measures an individual on the three major dimensions of hardiness i.e. control, commitment and challenge. Scores from 0 to 9 indicates moderate hardiness while score below 0 indicates low hardy personality. The reliability of this scale is 0.628 and the validity is 0.543 as calculated by the test maker [20].

\section{Emotional Autonomy Scale (EAS)}

There are four components of emotional autonomy: Two relatively more cognitive components: 1) Perceives parents as people 2) De-idealization and two relatively more affective components: 3) Non-dependency on parents 4) Individuation. The items were based on the contemporary perspective that de-emphasizes the storm and stress of adolescent detachment, rebellion and conflict but lays more stress on the processes of individuation. A total of 20 items constitute the test and are presented in declarative statements. The internal consistency as determined by Cronbach's alpha is 0.75 [21].

\section{Beck scale for Suicide Ideation (BSSI)}

This scale is a self-report measure and has 19 items that assess suicidal ideations, planning and intent to commit suicide in the past week. It has a three-point Likert type scale from 0 to 2 and the range of score is from 0 to 38. If a subject gets a high score that shows that he has higher suicide ideations [22].

\section{RESULTS AND DISCUSSION}

In order to study the variables of depression, hopelessness, hardiness and emotional autonomy, as predictors of suicide ideation in adolescents, regression analysis was used. But prior to that, the four predictor variables, which are highly correlated, were subjected to factor analysis to obtain independent factors so as to remove the issue of multicollinearity which distorts the results of regression analysis. Table 1 shows the results of factor analysis with the four predictor variables.

Table No 1: Factor loadings of predictor variables on single factor

\begin{tabular}{|c|c|}
\hline VARIABLES & FACTOR 1 \\
\hline Hopelessness & .78 \\
\hline Depression & .84 \\
\hline Hardiness & -.64 \\
\hline Emotional autonomy & .50 \\
\hline Eigen Value & $\mathbf{2 . 0 0}$ \\
\hline \% variance & $\mathbf{5 0 . 0 0}$ \\
& \\
\hline
\end{tabular}

As seen in Table 1, the four variables of hopelessness, depression, hardiness and emotional autonomy form a single factor explaining $50 \%$ of variance. The eigen value of the factor is 2.00 . The factors have high 
significant loadings on hopelessness (.78), depression (.84) and emotional autonomy (.50), whereas hardiness shows a negative loading on the same factor (-.64).

We can decipher from the results of factor analysis that the predictor variables are associated with one another and form a single factor explaining $50 \%$ variance. The factor loads highest on depression followed by hopelessness. Hence, we can say that in the factor predicting suicide ideation, depression is a significant factor forming a cluster with other cognitive variables. Hopelessness also loads positively on same factor showing that higher depression leads to hopelessness or vice versa, the two being most important predictors of suicide ideation among adolescents. Depressed state of mind further leads to forming self-reinforcing negative thoughts and cognitions about self, the world and one's future which translates into feelings of hopelessness. Thus, the present results from factor analysis confirm the association of the two variables in predicting suicide ideation among adolescents. Previous literature also shows high positive association between depression and hopelessness [4-5].

Emotional autonomy is another cognitive variable which positively associates with depression and hopelessness in adolescents. Adolescent period is marked by great turmoil and stress, given the myriad changes occurring both at physical as well as psychological level. In such a time, emotional detachment from parents, loss of emotional bonds, increased sense of individuation and non-dependency on parents, all add up to negative cognitions like depression and hopelessness, which further leads to development of suicidal ideations among both male and female adolescents [13-14].

Hardiness as a personality dimension loads negatively on the same factor, thus we can label the same as a protective factor against development of depression, hopelessness and emotional autonomy in adolescents. Hardy individuals are more capable of fighting stressful situations with high control on their emotions. They stay committed to their goals despite of the difficulties they face. Moreover, they possess the capability to face challenges and fight negative emotions. Hence such individuals are less likely to develop negative emotions like depression or hopelessness in comparison to those individuals who are low on hardiness [17]. The factor scores for the factor obtained were computed in SPSS using Anderson-Rubin method which gives us independent scores. Using these scores as the predictor variable, linear regression was applied predicting suicide ideation in adolescents. The purpose for doing so was to remove the issue of multicollinearity that arises in regression when predictor variables are correlated to one another. Table 2 shows the results of linear regression showing the $\mathrm{R}^{2}$, Adjusted $\mathrm{R}^{2}$, Regression Coefficient and Standardized Regression Coefficient $(\beta)$.

Table No 2: Results from Linear Regression with Suicide Ideation as Criterion Variable

\begin{tabular}{|c|c|c|c|c|c|}
\hline Predictor Variable & $\mathbf{R}^{2}$ & Adjusted $\mathbf{R}^{2}$ & $\begin{array}{c}\text { Regression } \\
\text { Coefficient }\end{array}$ & $\boldsymbol{\beta}$ & $\begin{array}{c}\mathbf{p} \\
\text { value }\end{array}$ \\
\hline Factor 1 & .336 & .334 & 2.79 & .57 & 0.01 \\
\hline
\end{tabular}

As seen in Table 2, the factor obtained in prior analysis, significantly predicts suicide ideation among adolescents explaining almost $37 \%$ variance in suicide ideation. The regression coefficient is significant and positive $(2.79, p<0.01)$ showing that the composite factor having significant loadings on depression, hopelessness, emotional autonomy and hardiness, positively predicts suicide ideation in adolescents.

Results from regression analysis bring out the predictive power of a single factor in predicting suicide ideation among adolescence. The factor with high and positive loadings on depression, hopelessness and emotional autonomy, show that presence of these cognitive variables in adolescents, leads to higher suicide ideations among them. On the other hand, hardiness acts as a protective factor combating the negative effects of these emotions which further lead to developing thoughts about ending one's life. The presence of this one factor alone explains $37 \%$ variance in suicide ideations among adolescents.

Among the variables which are positive indicators of suicide ideation from factor 1, depression loads the highest, thus indicating that it is the most important and positive predictor of suicide ideation among adolescence. Adolescents high on depression, are more prone to developing negative cognitions like suicidal 
ideas etc. They lose capacity to reason and think positively, thus making them more vulnerable to harbour such ideas like killing oneself or planning of such acts. In previous research also, depression emerged as significant predictors of suicide ideations among adolescence [8]. Hopelessness is another strong predictor of suicide ideation, which has high loading on the factor. Along with depression, hopelessness emerges as a significant variable leading to suicide ideations. Several theories like The Hopelessness Theory [7] and Cognitive Distortion Theory [8], both highlight the strong role of hopelessness, in predicting suicide ideation in the adolescent population, who are at high risk for developing the same. The negative feelings about self and the world, further feed negative thoughts, thus predicting higher suicide ideation in those who have higher levels of hopelessness. Hence the presence of these two variables lead to development of suicidal thoughts in both male and female adolescents.

Emotional Autonomy is another variable forming a cluster with depression and hopelessness and further it predicts suicide ideation positively. Increase in feelings of emotional autonomy especially during adolescent stage has a negative effect on the adolescents. Because of the ongoing transitions they face during the phase, losing sense of emotional bond and attachment with parents can further lead to forming suicidal thoughts and behaviours. Parental support is crucial during the adolescent years in helping them deal with their issues. In absence of such a warm and cohesive environment at home, and strained relationships with parents, negative cognitions among adolescents are more likely to rise, suicide ideation being most important of these. Hence, emotional autonomy is another important cognitive variable contributing positively to suicide ideation among them. Previous researchers have reported similar findings highlighting the positive role of emotional autonomy contributing to suicide ideation in the adolescent population [13-15].

Hardiness loads negatively on the factor thus acting as a protective force against development of suicide ideations among adolescents. As hardiness combats depression, hopelessness and other negative states of one's life, it is less likely that hardy individuals will develop suicidal ideations or tendencies. Hardiness in adolescents helps them to deal with stressful situations in life. They successfully cope up with negative emotional states and hence do not develop suicidal thoughts. On the other hand, adolescents low on hardiness, and high on depression and hopelessness, are highly vulnerable to developing suicidal ideations. Prior research also confirms the positive role of hardiness I combating suicide ideations among adolescents [17].

The current research thus brings out the combined role of cognitive variables i.e. depression, hopelessness and emotional autonomy in predicting suicide ideation among adolescents. On the other hand, hardiness forms a protective factor against development of such negative cognitions.

\section{CONCLUSION}

The current study was conducted with an aim to explore predictors of suicide ideation among adolescents. Keeping in mind the existing literature, depression, hopelessness, emotional autonomy and hardiness were taken as predictors of suicide ideation in adolescent population. The variables were first factor analyzed revealing a single factor comprising of the predictor variables. The factor was further used in linear regression predicting suicide ideation. The regression coefficient was significant thereby indicating that these variables form a cluster and further significantly predict suicide ideation among adolescents. The study can further be extended using other cognitive and environmental variables contributing to suicide ideation in adolescents.

\section{REFERENCES}

1. Lerner RM, Galambos NL. Adolescent development: Challenges and opportunities for research, programs, and policies. Ann Rev Psychol 1998;49(1):413-46.

2. O'Carroll PW, Berman AL, Maris R, Moscicki E, Tanney B, Silverman M. Beyond the tower of Babel. In Suicide Prevention 2002 (pp. 23-39). Springer, Boston, MA.

3. Joiner TE. Why People Die by Suicide: further Development and Tests of the Interpersonal-Psychological Theory of Suicidal Behaviour. InFlorida State University: Florida; 2005.

4. Rhodes AE, Bethell J, Bondy SJ. Suicidality, depression, and mental health service use in Canada. The Can J Psychiatry 2006;51(1):35-41.

5. Chioqueta AP, Stiles TC. Personality traits and the development of depression, hopelessness, and suicide ideation. Personal Individ Diff 2005;38(6):1283-91. 
6. Goodenow C. The psychological sense of school membership among adolescents: Scale development and educational correlates. Psychol Schools 1993;30(1):79-90.

7. Abramson LY, Metalsky GI, Alloy LB. Hopelessness depression: A theory-based subtype of depression. Psychol Rev 1989;96(2):358-80.

8. Palmer EJ, Connelly R. Depression, hopelessness and suicide ideation among vulnerable prisoners. Crim Behav Ment Health 2005;15(3):164-70.

9. Beck AT. Depression: Clinical, experimental, and theoretical aspects. University of Pennsylvania Press; 1967.

10. Nock MK, Kazdin AE. Examination of affective, cognitive, and behavioral factors and suicide-related outcomes in children and young adolescents. J Clin Child Adolesc Psychol 2002;31(1):48-58.

11. Crittenden PM. Internal representational models of attachment relationships. Infant Ment Health $\mathrm{J}$ 1990;11(3):259-77.

12. Steinberg L, Silverberg SB. The vicissitudes of autonomy in early adolescence. Child Dev 1986;2:841-51.

13. Frank SJ, Avery CB, Laman MS. Young adults' perceptions of their relationships with their parents: Individual differences in connectedness, competence, and emotional autonomy. Dev Psychol 1988;24(5):729-39.

14. Coco AL, Pace U, Zappulla C. Autonomia emotiva in adolescenza e benessere psicologico. Età Evolutiva 2000:76-81.

15. Pace U, Zappulla C. Relations between suicidal ideation, depression, and emotional autonomy from parents in adolescence. J Child Fam Stud 2010;19(6):747-56.

16. Kobasa SC. Stressful life events, personality, and health: an inquiry into hardiness. J Personal Soc Psychol 1979;37(1):1-10.

17. Erbes CR, Arbisi PA, Kehle SM, Ferrier-Auerbach AG, Barry RA, Polusny MA. The distinctiveness of hardiness, positive emotionality, and negative emotionality in National Guard soldiers. J Res Personality 2011;45(5):508-12.

18. Beck AT, Steer RA, Brown GK. Beck depression inventory-II. San Antonio 1996.

19. Beck AT, Weissman A, Lester D, Trexler L. The measurement of pessimism: the hopelessness scale. J Consult Clin Psychol 1974;42(6):861-9.

20. Maddi SR, Khoshaba DM. Personal views survey. Hardiness Institute; 2001.

21. Schmitz MF, Baer JC. The vicissitudes of measurement: A confirmatory factor analysis of the emotional autonomy scale. Child Dev 2001;72(1):207-19.

22. Beck AT, Steer RA, Ranieri WF. Scale for suicide ideation: Psychometric properties of a self- report version. J Clin Psychol 1988;44(4):499-505.

$* * * * * * * * * * * * * * * * * * * * * * * * * * * * * * * * * * * *$

Acknowledgements - Nil

Conflict of Interest - Nil

Funding - Nil 